\title{
Progressive neurodegenerative syndrome associated with Langerhans cell histiocytosis: a rare condition that we have to consider in patients with sporadic spastic ataxia and diabetes insipidus
}

\author{
Antonio Federico - Lara Di Toro Mammarella
}

Published online: 9 May 2012

(C) Springer-Verlag 2012

In this issue, Spagnolo et al. described a case of 58-yearold adult presenting Langerhans cell histiocytosis ( $\mathrm{LCH})$ pathology, in which psychic changes, dysarthria, dysphagia, opsoclonus, dysmetria and tremor were associated. MRI detected bilateral T2-hyperintense alterations within the dentate nuclei and adjacent white matter involving the middle cerebellar peduncles. In the past medical history, at the age of 38, diabetes insipidus was diagnosed and treated with desmopressin with a good response [1].

This report focuses the attention on the neurodegeneration in the course of LCH, mainly characterized by ataxia. In an interesting article, Wnorowski et al. collected 83 $\mathrm{LCH}$ cases, $25 \%$ of them presenting with neurological disorders (tremor, cerebellar ataxia, dysarthria, dysdiadochokinesia, psychomotor retardation and psychosis), with onset 3-15 years after initial LCH diagnosis.

The immunopathological pathogenesis of LCH suggests that this condition may be considered as a paraneoplastic disorder of the CNS, in which cerebellum is a special target [2].

Very often, contrary to this case in which neurodegeneration was detected after the $\mathrm{LCH}$ diagnosis, patients may have an infantile $\mathrm{LCH}$ onset with minimal signs followed by a slow progressive neurological disorder mimicking a neurodegenerative disease. A delayed diagnosis was reported by Jarquin-Valdivia and Buchhalter [3] in 2001 in

A. Federico $(\varangle) \cdot$ L. Di Toro Mammarella Department of Neurological Neurosurgical and Behavioural Sciences, Medical School, University of Siena, Viale Bracci 2, 53100 Siena, Italy

e-mail: federico@unisi.it a boy who presented with primarily neurological and endocrinological findings without pain, from a series of LCH paediatric patients (65) seen at Mayo Clinic.

As an example of this, we here report a 29-year-old male recently referred to us for progressive gait disturbances and dysarthria.

He was born of non-consanguineous parents, without a family history of similar disorders. At the age of 6 , he was affected by diabetes insipidus and treated with desmopressin. After some years, at the age of 18, 12 years after the onset of diabetes insipidus, he developed progressive spastic cerebellar ataxia.

Our neurological examination showed short stature, dysarthria, spastic ataxia, hyperactive deep tendon reflex and right extensor plantar response. Tremor, dystonia, memory disturbances, sensory or extrapyramidal symptoms or bone pain were absent.

He was a heavy smoker; respiratory symptoms were absent and he never performed lung analysis.

Routine blood tests were unremarkable. ACTH, GH, FSH, LH, cortisol and testosterone levels and thyroid functions were within normal limits.

Electrophysiological investigation was normal. Mild non-specific abnormalities were present in the muscle biopsy performed in the hypothesis of a mitochondrial disease. Bone lesions were not evident.

The brain TC showed calcifications in the cerebellar dentate nuclei (Fig. 1). Conventional brain MRI detected diffuse cerebellar and brainstem atrophy associated with bilateral T2-hyperintense signal alterations within the dentate nuclei. These findings, in association with the evidence of diabetes insipidus in the history, suggested the hypothesis of LCH neurodegeneration.

A high-resolution chest CT scan confirmed this hypothesis showing multiple cystic structures with a 


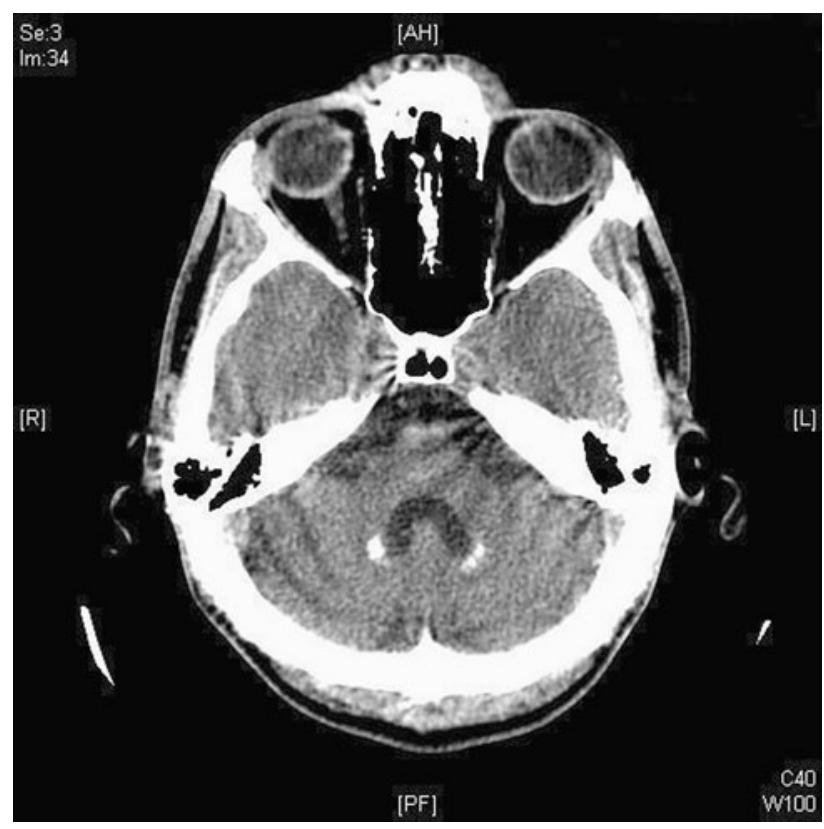

Fig. 1 Brain TC: calcification in dentate nuclei

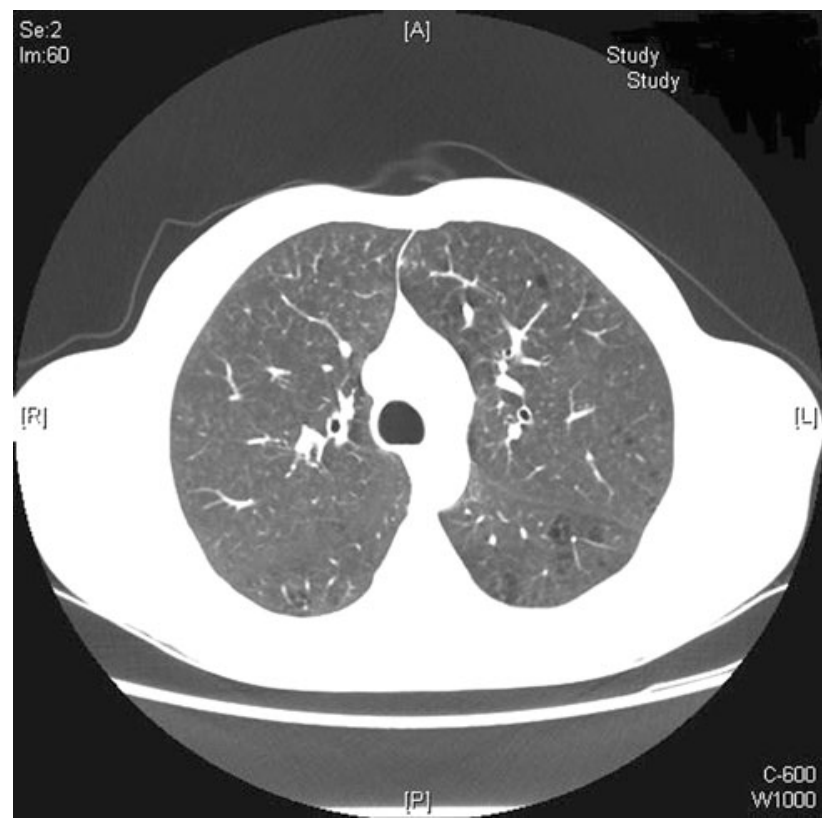

Fig. 2 High-resolution chest CT scan: multiple cystic structures with a typically thin wall typically thin wall predominating in the upper lobes (Fig. 2). A further confirmation was given by bronchoalveolar lavage fluid (BAL) analysis showing increased $\mathrm{CD} 1 \mathrm{a}+$ cells, a finding that almost exclusively occurs in $\mathrm{LCH}$.

In conclusion, we would like to stress that in the diagnosis of spastic paraparesis and ataxias, we have to consider the possibility of $\mathrm{LCH}$, mainly in sporadic cases, with diabetes insipidus in the clinical history, even without any evident LCH body manifestations, which we can investigate with deeper examinations.

\section{References}

1. Spagnolo F, Leopizzi E, Cardamone R, Falautano M, Martinelli V, Comi G, Volonté MA (2012) Neurodegeneration in the course of Langerhans cell histiocytosis. Neurol Sci. doi:10.1007/s10072011-0677-2

2. Wnorowski M, Prosch H, Prayer D, Janssen G, Gadner H, Grois N (2008) Pattern and course of neurodegeneration in Langerhans cell histiocytosis. J Pediatr 153(1):127-132

3. Jarquin-Valdivia AA, Buchhalter J (2001) Delayed diagnosis of a pediatric Langerhans' cell histiocytosis: case report and retrospective review of pediatric cases seen at Mayo Clinic. J Child Neurol 16(7):535-538 